\title{
Cambios recientes en la mortalidad por cáncer de próstata en España: estudio de tendencias en el período 1991-2005
}

\author{
Cayuela A*, Rodríguez-Domínguez $\mathrm{S}^{* *}$, Vigil Martín $\mathrm{E}^{*}$, Barrero Candau $\mathrm{R}^{* * *}$. \\ *Servicio de Documentación Clínica. Hospitales Universitarios Virgen del Rocío. Sevilla. \\ **Centro de Salud Pino Montano. Sevilla. \\ ***Servicio de Urología. Hospitales Universitarios Virgen del Rocío. Sevilla.
}

Actas Urol Esp. 2008;32(2):184-189

\section{RESUMEN}

CAMBIOS RECIENTES EN LA MORTALIDAD POR CÁNCER DE PRÓSTATA EN ESPAÑA: ESTUDIO DE TENDENCIAS EN EL PERÍODO 1991-2005

Objetivo: Describir la evolución de la mortalidad por cáncer de próstata en España en el periodo 19912005.

Sujetos y métodos: Realizamos un análisis de tendencia de las tasas estandarizadas de mortalidad (población estándar Europea) mediante análisis de regresión "joinpoint", el cual nos permite estimar el porcentaje de cambio anual de las tasas y localizar cambios significativos en la tendencia.

Resultados: Las tasas de mortalidad estandarizada alcanza su máximo valor en 1996. El análisis "joinpoint" identifica dos periodos diferentes en la tendencia de las tasas estandarizadas: uno inicial de incremento entre 1991 y 1996 ( $2,1 \%$ de incremento annual) y un segúndo periodo comenzando en 1996, en el que las tasas descienden un $-2,7 \%$ anual.

Conclusión: La tendencia de la mortalidad por cancer de prostata en España es similar a la de otras áreas industrializadas, con un incremento en las tasas estandarizadas que sufren un descenso hacia el final de la década pasada. El descenso en la mortalidad por cáncer de próstata es ahora evidente en 14 de las 17 Comunidades Autónomas. El incremento del cribaje oportunistico con PSA y una mejora en el tratamiento, posiblemente actuando en combinación, constituye la hipótesis más plausible.

Palabras clave: Cáncer de próstata. Mortalidad. Tendencia. Epidemiología.

\section{ABSTRACT \\ RECENT CHANGES IN PROSTATE CANCER MORTALITY IN SPAIN. TRENDS ANALYSIS FROM 1991 TO 2005}

Objetive: To describe the evolution of prostate cancer mortality in Spain during the period 1991-2005.

Subjects and methods: A trend analysis for age standardized mortality rates (European standard population) was performed, using joinpoint regression analysis, which allows estimation of the annual percent change of rates and to find significant changes in such trend.

Results: Age standardized mortality rates in Spain reached their peak value in 1996. The joinpoint analysis identified two different periods in the trend of the age standardized rates: a first one of increase in rates between 1991 and 1996 (2.1\% annual increase) and a second period starting in 1996, in which rates decline at an annual rate of $2.7 \%$.

Conclusion: The tendency of prostate cancer seen in Spain resembles that of industrialized areas, with an increase in its age standardized death rates that suffers a downturn by the end of the past decade. Mortality declines for prostate cancer are now evident in 14 out of the 17 Autonomous Communities. Increases in PSA screening and better treatment of early-stage disease, possibly acting in combination, remain plausible hypotheses.

Keywords: Prostate cancer. Mortality trends. Epidemiology. 
A nivel mundial, el cáncer de próstata (CP) es el segundo cáncer más frecuente en varones con una tasa de incidencia de 25,3 por $100000^{1}$.

En los países de la Unión Europea (UE) el CP es la forma más frecuente de cáncer en los hombres, con 301500 casos nuevos $(24,1 \%$ de todos los casos incidentes) seguido por el cáncer de pulmón (194.400 casos, 15,5\%) y el cáncer colorectal (163.100 casos, 13,0\%). La estimación de la incidencia del CP, para el año 2006, en la UE oscila entre 182 y 51 casos por 100.000 varones en Irlanda y Polonia respectivamente. Para España la incidencia estimada es de 77,2 casos por 100.000 varones nos posiciona en una situación intermedia baja en el contexto de la $\mathrm{UE}^{2}$. La incidencia se ha visto incrementada en las últimas décadas, debido principalmente a la extensión del uso de procedimientos de detección precoz ${ }^{1}$.

En la UE en el periodo 1997-2002 se observa un descenso en las tasas de mortalidad por CP del -1,4\% anual ${ }^{3}$. En España, las tasas de mortalidad por CP aumentan en el periodo 19511970, momento en el que las tasas alcanza una meseta y permanecen estables hasta que a mediados de los noventa comienzan a descender $^{4-7}$. En un análisis del periodo 1975-2000 se observó que ese descenso afectaba a las Comunidades Autónomas de Baleares, Valencia, Cataluña y Madrid, mientras que en las restantes la tendencia continuó siendo ascendente ${ }^{8}$. Recientemente se ha observado también este descenso en la Comunidad Autónoma de Andalucía en el periodo $1975-2004^{9}$.

Teniendo en cuenta todo lo anterior nos planteamos el objetivo de proporcionar información actualizada y analizar los cambios recientes en la tendencia de la mortalidad por cáncer de próstata en España en el período 1991-2005 usando modelos de regresión "joinpoint" y verificar si el descenso observado en estudios previos continúa y se ha extendido a otras Comunidades Autónomas.

\section{MATERIAL Y MÉTODO}

Los datos de mortalidad se han obtenido de la base de datos del Instituto Nacional de Estadística (INE), que recoge a partir de los certificados de defunción y siguiendo los criterios de las diferentes Revisiones de la Clasificación Internacional de
Enfermedades y Causas de Muerte (CIE), dichos datos por causa, sexo, edad y lugar de residencia ${ }^{10}$. Se han usado las defunciones por cáncer de próstata (código 185 de la $9^{\mathrm{a}}$ revisión de la CIE y código C61 de la $10^{\text {a }}$ revisión de la CIE para los periodos 1991-1998 y 1999-2005 respectivamente).

Como denominadores, se han utilizado las estimaciones de población a 1 de julio de cada año, por grupos de edad, facilitadas por el $\mathrm{INE}^{10}$. Se han calculado las tasas estandarizadas global por el método directo (usando como estándar la población europea) expresadas como tasas por 100.000 varones-año.

Para el análisis de tendencias de las tasas estandarizadas se utilizó modelos de regresión "joinpoint". El resultado de estos modelos es doble: identifican el momento en que se producen los cambios significativos de la tendencia y estiman la magnitud del aumento o el descenso observado en cada intervalo. Para la estimación de dichos modelos se utilizaron las tasas estandarizadas de mortalidad y sus errores estándar, y para los modelos con tasas específicas por edad se utilizaron las tasas y las poblaciones bajo un modelo de distribución de Poisson. Nuestra experiencia previa nos ha demostrado que la tendencia lineal final puede verse muy influenciada por variaciones en algunos de los datos extremos. Dado que queríamos reducir la posibilidad de tendencias descendentes que fueran simplemente el resultado de una fluctuación aleatoria en los datos, fijamos el mínimo número de datos en la tendencia lineal en ambos extremos del periodo en 5. Se buscó un máximo de dos puntos de inflexión en cada regresión, para lo cual el programa busca el modelo más sencillo que se ajuste a los datos mediante la técnica de mínimos cuadrados ponderados, estimando luego su significación estadística por medio de permutaciones Monte Carlo. La significación estadística se fijó en el $0,05 \%{ }^{11}$.

El cálculo de las tasas de mortalidad y sus errores estándar fue realizado en una hoja de cálculo (Microsoft ${ }^{\circledR}$ Excel). Los análisis "joinpoint" se realizaron mediante el software 'Joinpoint' version 3.0 del Surveillance Research Program of the US National Cancer Institute ${ }^{12}$.

\section{RESULTADOS}

Si nos atenemos al número de defunciones por CP, observamos un incremento del $29,9 \%$ a lo 
largo del período 1991-1998 (de 4.410 defunciones en 1991 a 5.728 en 1998), seguido de un descenso del $-3,8 \%$ en el periodo 1998-2005. En 2005, el CP se sitúa en tercer lugar como causa de muerte por cáncer en los varones (5.511 defunciones, $8,8 \%$ del total de tumores) detrás del cáncer de pulmón (16.645 defunciones, $26,6 \%)$ y del colon-rectal (7.472 defunciones, $11,9 \%)$.

En la Figura 1 se muestran las tasas (brutas y estandarizadas) de mortalidad por cáncer de próstata en España en el periodo 1991-2005. Las tasas brutas de mortalidad se incrementan $(0.7 \%$ anual; no significativo) pasando de 23,1 defunciones por 100.000 varones-año en 1991 a 25,9 en 2005.

La Tabla 1 muestra los resultados del análisis de regresión "joinpoint", es decir, los puntos en los que las tasas (específicas por grupos de edad, brutas y estandarizadas) cambian significativamente y el porcentaje de cambio anual de cada tendencia a nivel de España. Por grupos de edad, la regresión joinpoint realizada con modelo Poisson muestra una tendencia constante al descenso (grupos de 45-54 años y 55-64 años) o estabilización en las tasas (grupo de 35-44 años), por debajo de los 65 años. Por encima de esa edad se observan dos periodos: uno inicial (19911996/8) de estabilización (grupos de 65-74 años y 75-84 años) o incremento (grupo de mayores de 85 años) seguido de otro de descenso estadística-

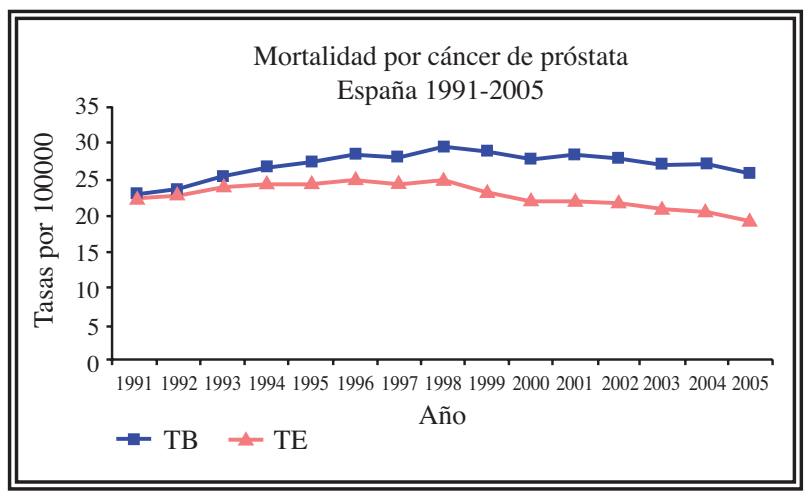

FIGURA 1. Tasas de mortalidad por cáncer de próstata (por cien mil varones-año). España 1991-2005. [TB: Tasa Bruta; TE: Tasa estandarizada (población europea)].

mente significativo. En relación a las tasas brutas se observan dos periodos: 1991-1998 (incremento porcentual del 3,2\%; $\mathrm{p}<0,05)$ y $1998-2005$ (descenso del $-1,7 \%$ anual; $\mathrm{p}<0,05$ ).

Las tasas ajustadas (globales) por edad en España descienden de 22,7 a 19,4 defunciones por 100.000 varones-año entre los años extremos del periodo estudiado (PCA: $-1,3 \% ; \mathrm{p}<0,05$ ). Sin embargo el análisis joinpoint refleja dos periodos estadísticamente significativos: 1991-1996 (incremento del $2,1 \%$ anual; $\mathrm{p}<0,05)$ y $1996-2005$ (descenso del $-2,7 \%$ anual; $\mathrm{p}<0,05$ ) (Tabla 1). Esta disminución afecta a todas las Comunidades Autónomas excepto Cantabria, Ceuta y Melilla, Extremadura y la Rioja en las que las tasas permanecen estables durante todo el periodo de estudio (Tabla 2). Dentro de España, la

Tabla 1: Análisis joinpoint de las tasas de mortalidad por cáncer de próstata en España (1991-2005)

\begin{tabular}{|c|c|c|c|c|c|c|c|}
\hline \multirow[b]{2}{*}{$\begin{array}{l}\text { Grupos de } \\
\text { edad }\end{array}$} & \multirow[b]{2}{*}{$\begin{array}{l}\text { Tasa } \\
1991\end{array}$} & \multirow[b]{2}{*}{$\begin{array}{l}\text { Tasa } \\
2005\end{array}$} & \multirow[b]{2}{*}{$\begin{array}{l}\text { PCA } \\
\text { global }\end{array}$} & \multicolumn{2}{|c|}{ Tendencia 1} & \multicolumn{2}{|c|}{ Tendencia 2} \\
\hline & & & & Periodo 1 & PCA & Periodo 2 & PCA \\
\hline $35-44$ & 0,2 & 0,1 & $-5,1$ & 1991-2005 & $-5,1$ & & \\
\hline $45-54$ & 2,0 & 2,0 & $-1,7 *$ & 1991-2005 & $-1,7 *$ & & \\
\hline $55-64$ & 16,1 & 12,4 & $-1,9 *$ & 1991-2005 & $-1,9$ * & & \\
\hline $65-74$ & 84,8 & 63,4 & $-2,1 *$ & $1991-1996$ & $-0,6$ & $1996-2005$ & $-2,6$ * \\
\hline $75-84$ & 292,7 & 230,8 & $-2,2 *$ & $1991-1996$ & 0,7 & $1996-2005$ & $-3,3$ * \\
\hline $85+$ & 594,3 & 638,9 & 0,3 & $1991-1998$ & 4,4 * & $1998-2005$ & $-3,0$ * \\
\hline TB & 16,9 & 25,9 & 0,7 & $1991-1998$ & 3,2 * & $1998-2005$ & $-1,7$ * \\
\hline $\mathrm{TE}$ & 22,7 & 19,4 & $-1,3^{*}$ & $1991-1996$ & $2,1^{*}$ & $1996-2005$ & $-2,7^{*}$ \\
\hline
\end{tabular}

TB: Tasa bruta por 100.000 varones-año

TE: Tasa estandarizada por 100000 varones-año (población estándar europea)

PCA: porcentaje de cambio anual estimado mediante análisis joinpoint

PCA global: PCA entre 1991 y 2005

${ }^{*}$ El PCA es significativamente distinto de $0(\mathrm{p}<0,05)$ 
Tabla 2: Análisis joinpoint de las tasas de mortalidad estandarizadas (población estándar europea) por cáncer de próstata en las Comunidades Autónomas de España (1991-2005).

\begin{tabular}{|c|c|c|c|c|c|c|c|}
\hline \multirow[b]{2}{*}{ Comunidad Autónoma } & \multirow[b]{2}{*}{$\begin{array}{c}\text { Tasa } \\
1991\end{array}$} & \multirow[b]{2}{*}{$\begin{array}{l}\text { Tasa } \\
2005\end{array}$} & \multirow[b]{2}{*}{$\begin{array}{c}\text { PCA } \\
\text { global }\end{array}$} & \multicolumn{2}{|c|}{ Tendencia 1} & \multicolumn{2}{|c|}{ Tendencia 2} \\
\hline & & & & Periodo 1 & PCA & Periodo 2 & PCA \\
\hline Andalucía & 19,5 & 19,3 & $-0,6$ & $1991-1996$ & 2,3 & $1996-2005$ & $-1,7^{*}$ \\
\hline Aragón & 24,6 & 20,0 & $-1,4^{*}$ & $1991-2000$ & 0,5 & $2000-2005$ & $-5,5^{*}$ \\
\hline Asturias (Principado de) & 20,9 & 23,9 & $-0,9$ & $1991-1996$ & 5,7 & $1996-2005$ & $-3,3^{*}$ \\
\hline Balears (Illes) & 26,8 & 18,7 & $-2,1^{*}$ & $1991-2005$ & $-2,1^{*}$ & & \\
\hline Canarias & 28,4 & 18,8 & $-3,2^{*}$ & $1991-2005$ & $-3,2^{*}$ & & \\
\hline Cantabria & 23,0 & 20,7 & $-1,1$ & $1991-2005$ & $-1,1$ & & \\
\hline Castilla -La Mancha & 20,3 & 17,7 & $-1,1$ & $1991-2005$ & $-1,0$ * & & \\
\hline Castilla y León & 21,9 & 19,5 & $-1,0^{*}$ & $1991-1994$ & 5,9 & $1994-2005$ & $-1,9^{*}$ \\
\hline Cataluña & 25,4 & 18,3 & $-2,2^{*}$ & $1991-1997$ & $-0,03$ & $1997-2005$ & $-3,6^{*}$ \\
\hline Ceuta y Melilla & 13,1 & 5,5 & $-0,1$ & $1991-2005$ & $-0,1$ & & \\
\hline Comunidad Valenciana & 22,8 & 20,2 & $-1,0$ & $1991-1996$ & 3,9 & $1996-2005$ & $-2,9^{*}$ \\
\hline Extremadura & 21,7 & 18,4 & $-0,7$ & $1991-2005$ & $-0,7$ & & \\
\hline Galicia & 23,6 & 21,5 & $-0,4$ & $1991-2000$ & 0,9 & $2000-2005$ & $-3,2$ \\
\hline Madrid (Comunidad de) & 22,0 & 16,9 & $-2,4^{*}$ & $1991-1998$ & 0,1 & $1998-2005$ & $-4,7^{*}$ \\
\hline Murcia (Región de) & 22,5 & 18,9 & $-1,4^{*}$ & $1991-1998$ & $-1,3$ & $1998-2005$ & $-3,8^{*}$ \\
\hline Navarra (Comunidad Foral de) & 27,5 & 18,1 & $-2,8^{*}$ & $1991-2005$ & $-2,8^{*}$ & & \\
\hline País Vasco & 22,2 & 20,9 & $-0,7$ & $1991-1997$ & 1,8 & $1997-2005$ & $-2,1^{*}$ \\
\hline Rioja (La) & 26,8 & 23,5 & $-1,1$ & $1991-2005$ & $-1,1$ & & \\
\hline España & 22,7 & 19,4 & $-1,3^{*}$ & $1991-1996$ & $2,1^{*}$ & $1996-2005$ & $-2,7^{*}$ \\
\hline
\end{tabular}

PCA: porcentaje de cambio anual estimado mediante análisis joinpoint PCA global: PCA entre 1991 y 2005

* El PCA es significativamente distinto de $0(\mathrm{p}<0,05)$

mayor mortalidad por cáncer de próstata en el año 2005 se observa en Asturias y en la Rioja, con tasas ajustadas superiores a los 23 casos por 100.000 varones-año, seguidas de Galicia (Tabla 2). La mortalidad es menor en Ceuta-Melilla, Madrid y Castilla-La Mancha (tasas inferiores a los 18 casos 100.000 varones-año).

\section{DISCUSIÓN}

Las posibles limitaciones de nuestro estudio surgen de las peculiaridades de la fuente de información (certificado de defunción) y del fenómeno que se evalúa (mortalidad). Pese a ello, la medida del estado de salud de una población, más útil y más ampliamente utilizada, continúa siendo la causa de muerte obtenida en los certificados de defunción ${ }^{13}$. Dada la escasa disponibilidad de datos sobre morbilidad, la mortalidad es el único indicador con carácter universal disponible en nuestro país. Los problemas relaciona- dos con la codificación y la certificación de la causa de muerte se deben tener en cuenta al explicar las tendencias de mortalidad observadas, aunque la precisión de los datos de mortalidad es considerable en el caso del cáncer ${ }^{14,15}$.

Para tener en cuenta el incremento de población observado en España durante el periodo de estudio utilizamos las tasas brutas por 100.000 varones y vemos que éstas tras un periodo de incremento comienzan a descender (Tabla 1). Hemos de tener en cuenta además que la población española ha envejecido y que el riesgo de morir por CP aumenta exponencialmente con la edad (Tabla 1, Fig. 2). Por ello, al analizar las tasas estandarizadas (Tabla 1 y Fig. 1), una vez controlado el efecto del incremento y envejecimiento de la población que lógicamente tiende a elevar estas cifras, vemos que la mortalidad por $\mathrm{CP}$ en España sufre un marcado descenso durante el período 1996-2005. La importancia de ese 
descenso es realzada por su magnitud (las tasas ajustadas descienden en dicho periodo un $-2,7 \%$ ) y por la relevancia del $\mathrm{CP}$ como problema de salud pública (es la tercera causa de muerte por tumores en España, ocasionando el 8.8\% de la misma). Para comprender las causas de dicho descenso sería necesario conocer las tasas de incidencia y de letalidad de CP en España durante este período.

Actualmente los datos sobre incidencia se obtienen a partir de estimaciones realizadas con los datos de los registros de base poblacional existentes. En ellos, se ha observado un incremento en la incidencia de $\mathrm{CP}$ que ha sido atribuida a: el aumento de la esperanza de vida (con el consiguiente aumento de la edad poblacional), la utilización, desde finales de los años 80 , del antígeno prostático específico (PSA) que permite el diagnóstico en fase preclínica aumentando así el número de casos diagnosticados, y la mejora y extensión de los métodos diagnósticos de imagen. Pese a ello, dada la escasa cobertura actual de éstos, se desconoce su incidencia y tendencia real.

Nuestros resultados (Tabla 1) muestran una tendencia descendente en las tasas de mortalidad en casi todos los grupos de edad a lo largo del periodo de estudio lo que sugiere un efecto periodo (debido a factores que afectan a gran parte de la población y que muestran sus consecuencias tras un corto período de latencia), descrito también en otras áreas geográficas) ${ }^{16}$. Las mejoras a nivel de diagnóstico y tratamiento estarian entre los posibles candidatos a esta explicación ${ }^{17,18}$. Así, en algunos estudios se ha hipotetizado sobre el efecto combinado de la detección precoz mediante la utilización del antígeno prostático especifico (PSA) y la mejora del tratamiento en estadios tempranos de la enfermedad ${ }^{7,19}$. En España, el incremento de resecciones transuretrales, la detección precoz junto con el incremento de la tasa de prostatectomias radicales para enfermedad localizada que comenzó antes de la introducción del PSA y el incremento de la terapia hormonal que se produce al mismo tiempo que se introduce el PSA podrían haber jugado un papel relevante en dicho descenso. De hecho, la supervivencia para el CP de los casos diagnosticados en el periodo 1990-1994 fue del $86 \%$ al año y del $65 \%$ a los 5 años ${ }^{20}$, lo que supone un incremento del $3 \%$ y $11 \%$ respectivamente con respecto a los casos diagnosticados en el periodo 1985-1989²1.

Dado que el CP es más prevalente en varones de edad avanzada, que pueden tener varias comorbilidades, una ligera mejora en la supervivencia incrementará la probabilidad de que mueran por otra causa distinta al CP. Como consecuencia, las tasas de mortalidad por $\mathrm{CP}$ descenderán aunque no se observe un descenso en las tasas de incidencia.

En conclusión podemos afirmar que continúa el descenso observado en España en la mortalidad por cáncer de próstata y que éste se ha hecho evidente en 14 de las 17 Comunidades Autóomas. Poco sabemos sobre los determinantes del descenso y, por ello, se necesitan más estudios. Un mejor entendimiento de éstos es fundamental para planificar las estrategias de intervención más eficientes a fin de mantener el descenso en la mortalidad por CP en España ${ }^{22}$.

\section{REFERENCIAS}

1. Nelen V. Epidemiology of prostate cancer. Recent Results Cancer Res. 2007;175:1-8.

2. Ferlay J, Autier P, Boniol M, Heanue M, Colombet M, Boyle P. Estimates of the cancer incidence and mortality in Europe in 2006. Ann Oncol. 2007;18(3):581-592.

3. Levi F, Lucchini F, Negri E, La Vecchia C. Continuing declines in cancer mortality in the European Union. Ann Oncol. 2007;18(3):593-595.

4. Cayuela A, Lacalle JR, Perea E. Análisis de la mortalidad por cáncer de próstata. España 1951-1979. Oncología 1987;10(8):364-370.

5. Cayuela A, Lacalle JR, Gili M. Analysis of cohort mortality from prostatic cancer in Spain, 1951-1983. J Epidemiol Commun Health. 1989;43(3):249-252.

6. Bolumar F, Vioque J, Cayuela A. Changing mortality patterns for major cancers in Spain, 1951-1985. Int J Epidemiol. 1991;20(1):20-25.

7. Baade PD, Coory MD, Aitken JF. International trends in prostate-cancer mortality: the decrease is continuing and spreading. Cancer Causes and Control. 2004;15(3):237241.

8. López-Abente G, Pollán M, Aragonés N, Pérez B, Hernández V, Lope $\mathrm{V}$ et al. La situación del cancer en España. Ministerio de Sanidad y Consumo. Madrid, 2005.

9. Cayuela A, Rodríguez-Domínguez S, Vigil E, Medina R, Conde M. Tendencias divergentes en la mortalidad por cáncer de próstata en la Comunidad Autónoma de Andalucía (1975-2004) Actas Urol Esp. 2007;31(2):92-97.

10. http://www.ine.es/inebase/ último acceso 2/5/2007

11. Kim HJ, Fay MP, Feuer EJ, Midthune DN. Permutation test for joinpoint regression with applications to cancer rates. Statist Med. 2000;19(3):335-351. 
12. Joinpoint Regression Program, Versión 3.0. National Cancer Institute. Bethesda, EEUU disponible en: http://srab.cancer.gov/joinpoint/ultimo acceso 2/5/2007.

13. Sen K, Bonita R. Global health status: two steps forward, one step back. Lancet. 2000;356(9229):577-582.

14. Benavides FG, Bolumar F, Peris R. Quality of death certificate in Valencia, Spain. Am J Public Health. 1989;79(10): 1352-1354.

15. Pañella Noguera H, Thió CB, Rodríguez Sarmiento C, Roca Antonio J. Validación de la causa básica de defunción en Barcelona, 1985. Med Clin (Barc) 1989;92(4):129-134.

16. Potosky AL, Feuer EJ, Levin DL. Impact of screening on incidence and mortality of prostate cancer in the United States. Epidemiol Rev. 2001;23(1):181-186.

17. Hankey B, Feuer E, Clegg L, Legler J, Ries L, Hayes R et al. Cancer surveillance series: interpreting trends in prostate cancer-Part I: Evidence of the effects of screening in recent prostate cancer incidence, mortality and survival rates. J Natl Cancer Inst. 1999;91(12):1017-1024

18. Oliver S, May M, Gunnel D. International trends in prostate-cancer mortality in the "PSA era". Int $\mathrm{J}$ Cancer. 2001;92(6):893-898.
19. Oliver SE, Gunnel D, Donovan JL. Comparison of trends in prostate cancer mortality in England and Wales and the USA. The Lancet. 2000;355(9217):1788-1789.

20. Berrino, F., Capocaccia, R. y cols. (Eds.). Survival of cancer patients in Europe: the EUROCARE-3 Study. Ann Oncol. 2003;14(Supl. 5):1-118.

21. No authors listed. Survival of cancer patients in Europe. The EUROCARE-2 study. IARC Sci Publ. 1999;(151): 1572.

22. Granado de la Orden S, Saá C, Quintás A. Situación epidemiológica del cáncer de próstata en España. Actas Urol Esp 2006;30(6):574-582.

Correspondencia autor: Dr. A. Cayuela

Servicio de Documentación Clínica.

Hospitales Universitarios Virgen del Rocío.

Avenida de Manuel Siurot, s/n - 41013 Sevilla

Tel.: 955013299

E-mail autor: aurelio.cayuela.sspa@juntadeandalucia.es

Información artículo: Original - Cáncer de próstata

Trabajo recibido: mayo 2007

Trabajo aceptado: julio 2007 